\title{
Essentialism vis-à-vis Possibilia, Modal Logic, and Necessitism
}

\begin{abstract}
Pace Necessitism - roughly, the view that existence is not contingent-essential properties provide necessary conditions for the existence of objects. Sufficiency properties, by contrast, provide sufficient conditions, and individual essences provide necessary and sufficient conditions. This paper explains how these kinds of properties can be used to illuminate the ontological status of merely possible objects and to construct a respectable possibilist ontology. The paper also reviews two points of interaction between essentialism and modal logic. First, we will briefly see the challenge that arises against S4 from flexible essential properties; as well as the moves available to block it. After this, the emphasis is put on the Barcan Formula (BF), and on why it is problematic for essentialists. As we will see, Necessitism can accommodate both (BF) and essential properties. What necessitists cannot do at the same time is to continue to understanding essential properties as providing necessary conditions for the existence of individuals; against what might be for some a truism.
\end{abstract}

When reflecting about an object's existence conditions, one finds room-conceptual room, at least - for three kinds of properties: essential properties, sufficiency properties, and essentialand-sufficiency properties $(\S 1)$. Sufficiency properties are often appealed to in order to illuminate the notion of merely possible objects (i.e., possible-but-non-actual objects), as well as the ontological status of merely possible objects. Peacocke's theory is a salient contemporary example of one such application of sufficiency properties (\$2). Among plausible candidates for being essential properties, there are some-the popular flexible ones - that would threaten the validity of modal logic S4; more precisely, of any modal logic that validates the transitivity axiom, $\square \mathrm{p} \rightarrow \square \square \mathrm{p}$, characteristic of S4. There is room, however, for manoeuvre (§3). Essential properties are-perhaps unreflectively-often assumed to provide necessary conditions for the existence of objects. If we understand essential properties in this way, the existence of essential properties - whether flexible or not-is in tension with the truth-let alone validity - of the Barcan Formula, according to which, if it is possible that there is an $F$, then, there is something which is possibly an $F(\S 4)$. Necessitist views are committed to the Barcan Formula. Consequently, necessitists that would also believe in the existence of essential properties should offer a different understanding of essential property; and they do (§5). A claim that, despite being widely accepted, is denied by necessitists is that (actually) concrete entities could not exist without being concrete. Necessitists, together with their new understanding of essential property, however, can continue to asserting that (actually) concrete entities are essentially concrete (§5.2). 


\section{Essential and Sufficiency properties}

In this section, I will introduce, very briefly, the notions of essential property, sufficient-forexistence property and individual essence. In another survey for this journal, I have discussed at length the relations between these notions. Here, I will only call the reader's attention to those aspects that will be particularly relevant for the issues discussed in this paper.

When it comes to characterizing the notion of essential property, two typically opposed accounts are the Existential Modal Account (EMA) and the (Real-)Definitional Account (RDA). According to (EMA), the following biconditional characterizes what an essential property is: $P$ is an essential property of $a$ if and only if $a$ could not exist without being $P$. By contrast, only the left-to-right direction of that biconditional is true according to (RDA): If $P$ is an essential property of $a$, then $a$ could not exist without being $P$, but not the other way around. Let us assume that Socrates originated from $s$ and $e$; sperm and egg cell respectively. Despite the disagreement between them, both (EMA) and (RDA) agree that if the property of originating from $s$ and $e$ is essential to Socrates, then, Socrates cannot exist without that property. Both accounts, therefore, agree that essential properties provide necessary conditions for the existence of individuals. That essential properties provide necessary conditions is perhaps a truism for many. Yet, as we will see in $§ 5.2$, one should be willing to revise this claim at least to the same extent to which one grants the force of the arguments supporting Necessitism.

Some think that, in addition to essential properties, there are also sufficiency propertiesproperties which provide sufficient conditions for the existence of individuals. The very property of originating from $s$ and $e$ is often thought of as a plausible candidate for being a sufficiency property: the exemplification of that property would suffice for Socrates to exist. This means that, under the assumption that originating from $s$ and $e$ is indeed sufficient for Socrates to exist, there is no question that Socrates exists in all worlds where that property is exemplified, and exemplifies that property in all those worlds. For convenience, let us call ' $C$ ' the set of worlds where originating from $s$ and $e$ is exemplified.

Sufficiency properties need not be essential properties. If originating from $s$ and $e$ was a sufficiency property but not an essential one, Socrates would exist in all worlds belonging to $C$ but also in worlds outside $C$ where he would have different origins. Similarly, essential properties need not be sufficiency properties. If originating from $s$ and $e$ was an essential property but not a sufficiency one, Socrates would only exist in worlds that belong to $C$, but not in all of them. Despite this independence between essential and sufficiency properties, sufficiency properties can nonetheless be, in turn, essential properties. The notion of an individual essence is an essentialist notion that refers to those properties that are both essential to a given individual and sufficient for its existence. If originating from $s$ and $e$ was an individual essence of Socrates, $C$ would be the set of exactly those worlds where Socrates exists.

\section{Individual essences as a route to possibilia}

Individual essences open the route to legitimating talk of possibilia. 'Possibilia' (the plural of 'possibile') stands for possible individuals. Pragmatically, this might convey the presupposition that 'possibilia' is intended to exclude actual individuals, but it is not. Actual individuals are also possible individuals (as any actual truth is also a possible truth). To avoid confusion, it is standard in the literature to refer to possible-but-non-actual individuals by 'mere possibilia', and to continue to using 'possibilia' to refer to all possible objects (including the actual ones).

A key difficulty around mere possibilia is to understand their ontological status. A merely possible child of Wittgenstein is not around us, in the concrete realm-otherwise he would not be a merely possible child. So where is him, if anywhere? Does he exist? If so, can we 
refer (uniquely) to him? Could that very same mere possibile-agreedly non-concrete - be concrete (had Wittgenstein fathered him)? In general, it is not clear at all what mere possibilia are. I will divide this question into what Yagisawa calls 'the Specificity Problem' and what Peacocke calls 'the Actualization Puzzle' and show how individual essences can help in both cases.

\subsection{The Specificity Problem}

Plausibly enough, Wittgenstein - actually childless (or so let us assume) - could have had a child. Equally plausibly, he could have had a child with Betty Hutton. For those who endorse an ontology of (mere) possibilia, the truth of the sentence 'It is possible that Wittgenstein and Betty Hutton have a child (in common)' will easily imply the existence of a mere possibile; namely, a possible child of Wittgenstein and Betty Hutton. Let's consider an arbitrary possible child of theirs. This possible child is a subject of predication, and there are many things one might want to ask about "it". Is "it" six feet tall? Is "it" a female? Are there rather two of them: one six feet tall, and the other one female? Perhaps the one that is female is five feet tall but could have been six feet tall? Questions of this sort were formulated by Quineunder the assumption that there is no sensible answer to them-to raise suspicions about the ontology of possible objects. Yet, if we can avail ourselves of individual essences, one can either answer these questions - when answerable - or else illuminate why they are bad questions.

Let us assume that Socrates - an actual individual - is individuated by the property originating from $s$ and $e$; and, in general, that actual human beings are individuated by the corresponding sperms and egg cells. Take now a random sperm of Wittgenstein, $s^{*}$, and a random egg cell of Betty Hutton, $e^{*}$. It would seem natural to think that the property originating from $s^{*}$ and $e^{*}$ is equally individuative. It individuates a possible child of Wittgenstein and Betty Hutton. This is, roughly, Peacocke's elegant move to solve the Specificity Problem. He believes in individual essences (504). Furthermore, he believes - or at any rate assumes for the sake of discussion - that actual human beings are individuated by the sperms and egg cells they actually originated from. He further thinks that

It is wholly natural to apply the same principle to merely possible human beings. For each possible human being $\mathrm{x}$, it is possible that there is some sperm $\mathrm{y}$ and some egg cell $\mathrm{z}$ such that $x$ develops from y's fertilization of $z$. We can abbreviate this by $\diamond \exists y \exists z R x y z$. A merely possible human being is individuated by this relation $\mathrm{R}$ and the possible (or actual) objects $y$ and $z$ that verify the existential quantifications in $\diamond \exists y \exists z R x y z$. This is simply to extend to possible human beings the way in which actual human beings are individuated. (495)

According to Peacocke, the questions used above to motivate the Specificity Problem are presumably all bad questions (except the modal one, as we will see in $\S 2.2$ ). Mere possibilia are not concrete (504) and, therefore, none of them have properties that entail concretenessnor can they be concrete, as we will see in $\S 2.2$. Despite the fact that the above questions are not the right ones to motivate the Specificity Problem, the problem is nonetheless there. For, if we are to admit mere possibilia into our ontology, we'd better have answers to the identity and distinctness questions: Which are the individuative properties of mere possibilia by means of which we can refer to them? Because the properties used by Peacocke to derive mere possibilia are individuative (and therefore uniqueness) properties, there are ready answers to these questions. We could introduce the term ' $a$ ' to refer to that possibile that satisfies $\diamond R X s^{*} e^{*}$; and we know that there is only one such object, and that it is a possible child of Wittgenstein and Betty Hutton. (As Peacocke says (499), there is a parallel between how possibilia are derived in his account and how numbers might be said to be derivative entities as well.) 


\subsection{The Actualization Puzzle}

Using 'possibilia' to refer to all possible objects (including the actual ones) might have the advantage of suggesting that the most relevant difference (from a metaphysical point of view) between, for instance, an actual child of Obama and a merely possible child of him is that the former is actual while the latter is not. Their natures, however, would be similar in kind and, when expressible, expressible in similar terms. Under one interpretation of 'actual possible object' this is true of Peacocke's possibilism but, under another interpretation, this is false. The way Peacocke solves the Actualization Puzzle will help us understanding this difference.

The Actualization Puzzle has to do with the concreta/non-concreta impermeabilism thesis. Roughly, the thesis says that nothing concrete could be non-concrete, and nothing nonconcrete could be concrete. So the boundaries between these two realms are (necessarily) impermeable. We conceded above - and with good reason - that the mere possibile, let us call it ' $a$ ', individuated by $\diamond R x s^{*} e^{*}$ is non-concrete. Yet, part of what it means for something to be actualized - it would seem - implies that if $a$ had been actualized, it would have been concrete. However, an ontology of mere possibilia plus that understanding of what actualization involves would force us to deny the concreta/non-concreta impermeabilism thesis.

Peacocke's way out of the puzzle is to precisify how 'actualization' and 'being actual' should be understood in his possibilist framework. Roughly, for any individual essence, there is a non-concrete possibile. Those remain non-concrete (and necessarily so) whatever goes on in the concrete realm. Among them, there is $a$. And there is also the one corresponding to (concrete) Socrates; let us call it ' $b$ '. Between $a$ and $b$, only one is actual; namely $b$. But actual $b$ remains non-concrete. So what does it mean for possibile $b$ to be actual? It means that there is something satisfying $b$ 's individuating conditions; namely Socrates (505). Peacocke's ontology bag includes, therefore, all of the following. The actual and concrete Socrates; the actual and non-concrete Socrates-possibile; and the non-actual and non-concrete possibile $a$. The first one is concrete and is a contingent entity; the two last ones cannot be concrete.

Possibile $a$ is a possible child. Yet, $\ulcorner$ a possible $\mathrm{F}\urcorner$ is a predicate true ascriptions of which should not be understood as implying the modal property of possibly being an $F$. In particular, while $a$ is a possible child, $a$ could not be a child (505). This solution to the actualization puzzle helps us understanding why the modal question used in $\S 2.1$ to motivate the Specificity Problem is, according to Peacocke, a good question that should be answered in the negative. Non-concrete possibilia cannot have modal properties that entail the possibility of being concrete, since non-concrete possibilia are essentially, and necessarily, nonconcrete. This is a point of disagreement with the necessitist views discussed in $\S 5$.

In sum, if $S$ is the set of non-concrete possibilia that are possible humans, all of themwhether actual or not in that technical sense-are ontologically on a par; and their natures, when expressible, are all expressible in similar terms. If we also allow (actual and concrete) Socrates to qualify as a possible object - a possibile - it will no longer be true that all possible objects - whether actual or not - individuated by human-origin properties are similar in nature. Some are essentially concrete, whereas some are essentially non-concrete.

There is probably a lot more one might like to be told about this possibilist programme. For instance, will Peacocke also need a distinction — á la Zalta - between exemplification and encoding? Will Peacocke also apply similar distinctions to all abstract objects (thereby distinguishing, for instance, number-two from number-two-possibile?) If so, how are they different? If not, why should we believe in Socrates-possibile but not in number-twopossibile? The programme seems nonetheless to be doing the right steps towards dignifying a possibilist ontology. Bear in mind, however, that this requires a commitment to individual 
essences, and not everyone is ready to endorse them. ${ }^{1}$ Also, this strategy is best understood if one lets all properties of the kind $\diamond$ Rxyz be individuative properties and, if one were also inclined to think that some of these individual essences are flexible, this will imply a commitment to coincident entities of the same kind (to see why, see Roca-Royes 'Essential' $\S 4)^{2}$

\section{Flexible essential properties and S4}

According to essentiality of material origins for artefacts (EMOA), material origins are essential to artefacts. Let $m$ be the hunk of matter from which a given actual table, $a$, originated. According to strong - or inflexible-(EMOA), this would be an essential property of $a$ :

$F=$ being originally constructed from $m$.

Yet, strong (EMOA) seems to have intuitions against it. For intuition seems to tell us that the very same object (be this a table, a chair, a tree, a human being, etc.) could intuitively originate from slightly different origins. This intuition is accommodated by weak-or flexible-(EMOA). According to weak-(EMOA), $F_{m}$, and not $F$, is, in the actual world, an essential property of $a$ :

$F_{m}=$ being originally constructed from a hunk of matter overlapping to a high degree with $m$.

Salmon argued (Reference and Essence) that flexible essential properties like $F_{m}$ imply that the accessibility relation between worlds is not transitive. Given that the characteristic axiom of the modal logic S4 (namely, $\square \mathrm{p} \rightarrow \square \square \mathrm{p}$ ) requires that the accessibility relation be transitive, Salmon's argument from: flexible essential properties, to: the accessibility relation is not transitive, is also an argument from: flexible essential properties, to: $\mathrm{S} 4$ is not the correct modal logic. Despite the fact that Salmon's argument has been very influential, it rests on a rather controversial assumption. Let us first see the argument, and then the way it can be resisted.

Table $a$ originates from $m$ in the actual world, @. Let us assume that the flexible $F_{m}$ is essential to $a$. For simplicity, let us assume also that $m, m_{1}, m_{2}, m_{3}$ and $m_{4}$ are the only pieces of matter that overlap enough with $m$ for it to be possible that $a$ originates from any of them. Under this simplifying assumption, $F_{m}$ allows for $a$ to originate in some possible world, $w_{1}$, from $m_{3}$. Therefore-Salmon assumes - in $w_{1}$, where $a$ originates from $m_{3}$, $a$ 's essential property is $F_{m 3}$ (and not $F_{m}$; the one $a$ had essentially in the actual world, @):

$F_{m 3}=$ being originally made from a hunk of matter overlapping to a certain (high) degree with $m_{3}$.

Again for simplicity, let us assume that $m_{3}, m_{4} m_{5}, m_{6}$ and $m_{7}$ are the only pieces of matter that overlap enough with $m_{3}$. If this is so, then, there is a world, $w_{2}$, accessible from $w_{1}$, where $a$ originates from $m_{5}$. However, from $@$, it is not possible that $a$ originates from $m_{5}$. If ' $R$ ' stands for the accessibility relation, these are the accessibility facts of this scenario: $R @, w_{1}$,

\footnotetext{
${ }^{1}$ Those who do not believe in sufficient-for-existence properties-even though they might believe in essential ones-will not.

${ }^{2}$ Also Plantinga is well known for his individual-essence-based solution to the problem of mere possibilia. A salient difference between Plantinga's and Peacocke's is about the kind of individual essences they use. An individual essence is purely qualitative if it only involves individual-free properties and relations-like being taller than, or being human, and unlike being taller than John. It is purely non-qualitative, if it only involves individuals-like being Socrates. And it is neither purely qualitative nor purely non-qualitative, if it involves both-like originating from $\boldsymbol{s}$ and $\boldsymbol{e}$. Whereas Peacocke's individual essences are neither purely qualitative nor purely non-qualitative, Plantinga's are purely non-qualitative. An approach like Plantinga's is susceptible to difficulties that an approach like Peacocke's can — at least in principle_-avoid. See Menzel (and, in particular, the third problem under the section called 'Problems for Individual Essences') for an explanation of what these difficulties are.
} 
$R w_{1}, w_{2}$, but $\neg R @, w_{2}$. In other words, originating from $m_{5}$ is possibly possible for $a$ but not possible for $a$, and this goes against the transitivity of $R$.

This argument, however, can be resisted (Williamson Identity; and Roca-Royes 'Peacocke's') at the step where it is assumed that $F_{m 3}$ is, in $w_{1}$, essential to $a$. Instead, one might want to say - and insist against the argument's controversial assumption - that $a$ 's essential properties do not vary from world to world. If one goes this way, an option is to say that, in $w_{1}$-like in @-a's essential property is $F_{m}$ and not $F_{m 3}$, and that, consequently, there is no world, accessible from $w_{1}$, where $a$ originates from $m_{5}$. So $w_{2}$ does not exist. This being so, we are left with no counterexample to the transitivity of $R$.

This way of resisting Salmon's argument also blocks Lewis's complaint against Salmon's move and, importantly, it does so without renouncing flexible essential properties. While arguably not a friend of de re modality himself, ${ }^{3}$ Lewis points out a potential tension in Salmon's essentialist views. In particular, he points out that his assumption that an object could have (slightly) different essential properties in different worlds would easily amount to giving up essentialism altogether: "There is worse to come: couldn't we trace a very long chain of very small revisions leading from you to a poached egg?" (Lewis 244). This tension is avoided if the argument is resisted as above.

While some might disagree with Lewis on this potential tension and might also be ready to accept the non-transitivity of $R$, it is good news that Salmon's argument can be resisted in the way illustrated above. For many modal theorists believe that the accessibility relation among worlds is transitive but, at the same time, they also believe in flexible - rather than rigid-essential properties. Resisting the argument by denying the controversial assumption identified above shows, contra Salmon, that those two beliefs can consistently be simultaneously held. As a result, endorsement of flexible essential properties is not, per se, incompatible with S4.

\section{Essentialism and the Barcan Formula}

There are other problems that an essentialist will face, however, when reflecting on which modal logic and which semantics best models metaphysical modal truth. The problem to be discussed in this section, furthermore, arises independently of whether there are flexible essential properties or not. It arises from the fact that the simplest models-i.e., with nonvariable domains - of the fairly weak quantified modal logic $\mathrm{K}+\mathrm{CQT}^{4}$ validate the Barcan Formula (BF): ${ }^{5}$

(BF) $\forall \mathrm{x} \square \varphi(\mathrm{x}) \rightarrow \square \forall \mathrm{x} \varphi(\mathrm{x})$

(BF) is equivalent to $\diamond \exists \mathrm{x} \varphi(\mathrm{x}) \rightarrow \exists \mathrm{x} \diamond \varphi(\mathrm{x})$ and, for convenience, I will be discussing the natural reading of its equivalent formula.

The technical details should not matter for present purposes. It matters, however, to understand why (BF) is problematic for an essentialist. According to (BF), if it is possible that there is something that is $\varphi$, then, there is something that is possibly $\varphi$. Some brands of essentialism might have no problem with (BF). Extreme Haecceitism - the thesis that there are no essential properties - or P. Mackie's Minimalist Essentialism, by being very restrictive on what they count as essential properties, are correspondingly very liberal with respect to de

\footnotetext{
${ }^{3}$ Lewis can be classified as a sceptic about de re modality as a consequence of his inconstancy thesis $(\S 4.5)$, although this is subject to controversy (Divers, §4).

${ }^{4} \mathrm{~K}+\mathrm{CQT}$ results from adding the rules of Classical Quantification Theory (CQT) to the propositional modal logic K. (See Garson for further reading on this.)

${ }^{5}$ Despite (BF) being validated by these simple models, it is nonetheless not derivable in $\mathrm{K}+\mathrm{CQT}$. In order to be able to derive (BF), one would need to strengthen the logic. One could add (BF) itself as an axiom-as Linsky and Zalta do (1994) — or one could add further propositional modal axioms to get stronger modal logics, like B or $\mathrm{S} 5$, where $(\mathrm{BF})$ is derivable-like Williamson does.
} 
re modality. For these two sorts of essentialism, it is very easy to accommodate the truth of (BF). The extreme haecceitist-for whom there are no essential properties - needs only to believe in the existence of at least one actual object. For that very same actual object can be anything. (That she needs to believe in at least one object is due to the fact that the left hand side of the formula equivalent to (BF) that we are currently discussing does not commit us to the existence of any actual entity, but the right hand side - standardly understood - does.) Someone like P. Mackie-who endorses the concreta/abstracta impermeabilism thesis as one of the very few essentialist theses - needs only to believe - for similar reasons - in at least two actual objects: one abstract and one concrete. The more essential properties one believes in, however, the more difficult it is to believe in (BF).

Assume that Essentiality of Origins (EO) and Essentiality of Kind (SE) are, in some version applied to human beings, true. The former says that origins are essential to originated entities, and the later says that if $k-$ e.g., human being - is the fundamental kind of a given object, $o$ - e.g., Socrates - then $o$ is essentially of kind $k$. We know-or let us assume sothat Wittgenstein had no child. But he could have had one. In Symbols, $\diamond \exists x C(x)$, where the predicate ' $\mathrm{C}$ ' is interpreted as being a child of Wittgenstein. Contrary to (BF), however, and by the assumption that (EO) and (SE) are true, no actual individual is possibly a child of Wittgenstein; so $\neg \exists \mathrm{x} \vee \mathrm{C}(\mathrm{x})$. For, given (SE), any prima facie candidate must be found amongst actual human beings. Let us take an arbitrary human being, $c$. Such a human being has some actual origins. If $c$ were to be, in some world, $w$, a child of Wittgenstein, $c$ would have, in $w$, origins other than the actual ones. But this goes against (EO). Therefore, as soon as we are committed to two of the most popular essentialist principles-(EO) and (SE) - it seems that we need to deny $(\mathrm{BF})$.

Contrary to what this argument suggests, however, (BF) can consistently be endorsed together with essentialist principles like (EO) and (SE), and Necessitism offers an example of how this can be done.

Necessitism is, roughly, the thesis that existence is not contingent. Less roughly, according to Necessitism, all objects that there are necessarily exist, and there could not be more objects than the ones that there are. Necessitist theorists include Linsky, Zalta, and Williamson. To know where exactly these theorists' necessitist commitments come from is not strictly necessary for current purposes. ${ }^{6}$ What we are interested in here is to see how a necessitist can accommodate both essentialism and (BF).

To this effect, the first thing to explore is how necessitism stands vis-à-vis the assumption behind the Actualization Puzzle (seen in \$2.2). Necessitists will most probably grant that Wittgenstein and Betty Hutton could have had a child. By their lights, there is a possible child

\footnotetext{
${ }^{6}$ They come from the logics with which these theorists are working. As indicated in the previous footnote, necessitists like Linsky, Zalta and Williamson, work with $\mathrm{K}+\mathrm{CQT}$ strengthened in ways such that (BF) is derivable in the resulting logics. Now, Necessitism has two parts. First, all objects that there are necessarily exist and, second, there could not be more objects than the ones that there are. The first part is derivable in $\mathrm{K}+\mathrm{CQT}$. Roughly, from $\mathrm{K}+\mathrm{CQT}$, the Converse Barcan Formula (CBF), $\square \forall \mathrm{x} \varphi(\mathrm{x}) \rightarrow \forall \mathrm{x} \square \varphi(\mathrm{x})$, is derivable. In turn, (CBF), together with other theorems of K+CQT, implies (NE): $\forall \mathrm{x} \square \exists \mathrm{y} x=\mathrm{y}$. For, by taking $\varphi(\mathrm{x})$ to be $\exists \mathrm{y} x=\mathrm{y}-$ which is a theorem of CQT - we get the following instance of (CBF): ( $\forall \forall \mathrm{x} \exists \mathrm{y} x=y) \rightarrow(\forall \mathrm{x} \square \exists \mathrm{y} x=y)$. Both this formula and its antecedent are theorems of $\mathrm{K}+\mathrm{CQT}$ and, by Modus Ponens, so is therefore its consequent. Its consequent- $(\mathrm{NE})$ - is the thesis that all objects that there are necessarily exist. To get the second part, we need a stronger logic than $\mathrm{K}+\mathrm{CQT}$. Without strengthening it, we can derive (NNE) - $\square \forall \mathrm{x} \square \exists \mathrm{y} x=\mathrm{y}-$ by applying the Rule of Necessitation to (NE). Yet, (NNE) is still compatible with accessible worlds having increasingly bigger domains, and this is contrary to the second part of Necessitism. However, this is avoided if one works with the stronger logics with which the Necessitists work, where (BF) is derivable. For, as Williamson says ('Necessitism', §2), “(BF) holds if and only if there could not have been something which is not already something; there could not have been more than there is".
} 
of Wittgenstein and Betty Hutton and it exists necessarily. Unlike what we saw in discussing Peacocke's possibilism, this possible child of Wittgenstein and Betty Hutton can be a child of Wittgenstein and Betty Hutton. So Necessitism denies the concreta/non-concreta impermeabilism thesis. Every object exists necessarily, including you. The illusion that existence is contingent is replaced by necessitists by the surrogate that concreteness is contingent.

Because necessitists deny that impermeabilism thesis, they would quickly spot, in the argument above, an assumption they do not grant. The assumption is that any candidate to making ' $\exists \mathrm{x} \vee \mathrm{C}(\mathrm{x})$ ' true must be concrete. We indeed assumed this by assuming that any such candidate must be found amongst human beings; for human being entails concreteness. (This is to say that they deny that any such candidate must be a human being, and, more generally, that it must have any concreteness-entailing property.) In a nutshell, therefore, this is how a necessitist can accommodate the current potential counterexample to (BF): There is an object, $a$, that is not concrete, and that could be Wittgenstein's child.

The reader is probably puzzled. For it might seem obvious that if there is something such that: (i) it is not concrete (and therefore not human); but (ii) can be Wittgenstein's child (and therefore human), then, at least (SE) is false. Consequently - the reader might complain - this can hardly support the claim that (BF) can be consistently endorsed with (SE) and (EO).

The puzzlement is justified, and the sense in which (BF) is compatible with essentialism is still to be specified. We will see this in the next section, by means of making explicit two revisions that this view imposes.

\section{Necessitism and essential properties}

\subsection{Impermeabilism theses}

The first revision has already been mentioned above. Necessitists are committed to saying that a possible child of Wittgenstein is contingently non-concrete: it is non-concrete, but it could be concrete (since it could be a child of Wittgenstein). So necessitists will easily deny the widely accepted concreta/non-concreta impermeabilism thesis. (They surely will as soon as they believe in (EO) and (SE).)

This is not to say that necessitism denies also the concreta/abstracta impermeabilism thesis. Williamson has never denied this thesis - and indeed endorses it - and in the latest expressions of their views, Linsky and Zalta do not deny it either. ${ }^{7}$ To keep the concreta/abstracta impermeabilism thesis, it suffices to say that 'abstract' is not coextensional with 'non-concrete'; against, perhaps, what one might have thought prior to Necessitism. The difference might seem subtle, but it matters. Especially if one wants to remain as conservative as one can with respect to pre-necessitism treatments of (typically) abstract objects (see Zalta).

On what seems now to be common to the necessitist views one finds in the literature, therefore, the following is true. Number two is abstract, and necessarily so; Eiffel Tower is concrete, contingently so, but necessarily not abstract; and a possible child of Wittgenstein is non-concrete, contingently so, and necessarily not abstract. On this kind of necessitism, 'abstract' is co-extensional with 'necessarily non-concrete'. The exhaustive and exclusive distinction is therefore given by abstract and possibly concrete (or, extensionally equivalently, possibly non-concrete).

Whichever it is the impermeabilism thesis they deny, therefore, those necessitists that also have the essentialist intuitions supporting (EO) and (SE) will need to sacrifice at least some

\footnotetext{
${ }^{7}$ See Linsky and Zalta ('In defense of the contingently nonconcrete', 293fn.) and Zalta (678 and 685, where he says that "by definition, abstractness is equivalent to necessary nonconcreteness").
} 
other widely-shared essentialist intuitions; namely, the intuitions supporting the impermeabilist thesis denied. This is the first revision. We still need to specify the sense in which a necessitist can nonetheless continue to maintaining that a possible child of Wittgenstein is essentially human (and even essentially concrete, but not essentially nonconcrete!). More generally, we need to specify the sense in which the denial of the concreta/non-concreta impermeabilism thesis does not amount to endorsing Extreme Haecceitism - the thesis that there are no essential properties.

\subsection{Revisionism about (EMA) and (RDA)}

According to most essentialists, biological origins and fundamental kinds are essential. However, properties like being human or originating from $s$ and e entail concreteness. Therefore, if the latter is contingent of $a$, so must be the former. If they are contingent of $a$, however, they are not essential according to the way (EMA) and (RDA) characterize essential property. For, as seen in $\S 1$, according to both these views, if $P$ is essential to $a$, then $a$ could not exist without being $P$. Necessitists who are also essentialists, therefore, cannot understand essential properties in this way and, consequently, cannot take essential properties as providing necessary conditions for the existence of individuals. This is the second revision.

A preliminary question arises, however, as to whether, in order to capture essentialist intuitions, one will need to apply this revision to all objects - including the abstract ones - or only to the possibly concrete ones. For the revision is forced upon by the denial of the concreta/non-concreta impermeabilism thesis and, given that abstract entities are understood by current necessitists as being necessarily non-concrete, the denial of this impermeabilism thesis does not affect abstract entities.

An option for the necessitist is, therefore, to say that the notion of essential property that applies to the abstracta is not the same as the notion of essential property that applies to the possibly-concreta. This is, precisely, Zalta's position in his 'Essence and Modality' (678). He offers two independent characterisations of essential property: one for abstract objects, and one for possibly concrete objects. His view, therefore, offers the minimum revision on the characterisation of essential property that is required in order to accommodate essentialist intuitions in a (non-extreme-haecceitist) necessitist framework.

Given that the denial of the concreta/non-concreta impermeabilism thesis affects only possibly concrete objects, I will present only Zalta's notions of essential property that apply to possibly concrete entities, and identify both the sense in which this is revisionary and the reason why it can be said to accommodate essentialist intuitions.

Zalta distinguishes weakly-essential properties from strongly-essential ones (679). For any possibly concrete object, $x$ :

$P$ is weakly-essential to $x$ iff necessarily, if $x$ is concrete, then $x$ is $P$

$P$ is strongly-essential to $x$ iff (it is not necessary that $x$ is $P$ ) \& (necessarily, if $x$ is concrete, then $x$ is $P$ )

Of these two kinds of essential properties, the strongly-essential properties are the most adequate to answer the question 'what is $\mathrm{x}$ ?'. Without entering too much into the reasons why this is so, I shall only say that Fine's examples against (EMA) can easily be accommodated to argue that weakly-essential properties are not suitable to play this role. For instance, it is true that: necessarily, if Socrates is concrete, then Socrates is the sole member of $\{$ Socrates $\}$. On Zalta's definition, therefore, being the sole member of \{Socrates\} is weakly-essential to Socrates. Yet, it does not seem that this property illuminates what being Socrates consists in. ${ }^{8}$

\footnotetext{
${ }^{8}$ For more on this, see Fine 'Essence and Modality'.
} 
The characterization of strongly-essential property is revisionary with respect to both (EMA) and (RDA). For this characterization denies the left-to-right direction of (EMA) - the only one on which (EMA) and (RDA) agree - according to which if $a$ essentially Ps, then $a$ could not exist without $P$. By definition, $P$ being strongly-essential to $x$ entails that it is contingent whether $x$ is $P$. Therefore, not only is this characterisation opposed to (EMA) and (RDA), but also it is completely congenial to necessitism.

In which sense can this characterization accommodate essentialism? It is open to the necessitist to say that a possible child of Wittgenstein, $a$, is strongly-essentially human. Granted, this means that he can exist and yet fail to be human. However, provided that he is concrete, he cannot fail to be human. Similarly, it is open to the necessitist to say that originating from $s$ and $e$ is strongly-essential to $a$. That is, it is not necessary that $a$ originates from $s$ and $e$; however, necessarily, if $a$ is concrete, $a$ originates from $s$ and $e$. By testing the definition of strongly essential property with the properties concrete and non-concrete, the reader can verify that, as anticipated at the end of $\S 5.1$, only the former comes out as strongly essential. This is, roughly, the way in which (SE) and (EO) - and thereby the denial of extreme haecceitism — can be accommodated by necessitism.

To conclude, let me stress that Zalta's notions of weakly-essential property and stronglyessential property do not apply to abstract entities. For any abstract entity, $e$, no property $P$ is weakly-essential to $e$ or strongly-essential to $e$. For all this revision implies, therefore, number 2 continues to being essentially and necessarily the immediate successor (among integers) of number 1. By the same token, even when it is true that (it is not necessary that number 2 is green) \& (necessarily, if number 2 is concrete, then number 2 is green), the definition of strongly-essential property-by its being defined only for possibly concrete entities - does not allow us to say that being green is strongly essential to number 2.

\section{Acknowledgements}

My great thanks to Anand Vaidya, Dan López de Sa, Fabrice Correia, Genoveva Martí, Manuel García-Carpintero and Tuomas Tahko, for their helpful comments on earlier drafts of this paper. With special mention, I am greatly thankful and indebted to Gabriel Uzquiano and an anonymous referee for this journal, whose penetrating comments helped me to understand better the issues discussed here. I can only hope to have reflected all this help in the final version.

\section{Works Cited}

Fine, Kit. 'Essence and Modality'. Philosophical Perspectives 8 (1994): 1-16.

Garson, Jason. 'Modal Logic'. The Stanford Encyclopedia of Philosophy (Winter 2009 Edition), Edward N. Zalta (ed.), URL = $<$ http://plato.stanford.edu/archives/win2009/entries/logic-modal/>.

Lewis, David. On the Plurality of Worlds. Oxford: Basil Blackwell, 1986.

Linsky, Bernard and Zalta, Edward (1996). 'In Defense of the Contingently Nonconcrete'. Philosophical Studies 84 (1996): 283-294.

—. 'In Defense of the Simplest Quantified Modal Logic'. Philosophical Perspectives 8 (1994): 431-458.

Mackie, Penelope. How Things Might Have Been: Individuals, Kinds and Essential Properties. Oxford: Clarendon Press, 2006. 
Menzel, Christopher. 'Problems with the Actualist Accounts'. Supplement to 'Actualism'. The Stanford Encyclopedia of Philosophy (Summer 2010 Edition), Edward N. Zalta (ed.), URL = http://plato.stanford.edu/archives/sum2010/entries/actualism.

Peacocke, Christopher. 'Principles for Possibilia'. Noûs, 36/3 (2002): 486-508.

Plantinga, Alvin. The Nature of Necessity. Oxford: Oxford University Press, 1974.

Quine, Williard V.O. 'On What There Is'. Review of Metaphysics. Reprinted in Quine 1953: $1-19$.

Roca-Royes, Sonia. 'Essential Properties and Individual Essences'. Philosophy Compass, forthcoming.

—. 'Peacocke's Principle-Based account: Flexibility of Origins pus S4'. Erkenntnis 65/3 (2006): 405-426.

Salmon, Nathan. Reference and Essence, Princeton: Princeton UP, 1981.

Williamson, Timothy. 'Bare Possibilia'. Erkenntnis 48 (1998): 257-273.

—. Identity and Discrimination. Oxford: Basil Blackwell, 1990.

—. 'The Necessary Framework of Objects'. Topoi, 19 (2000): 201-208.

—. 'Necessitism, Contingentism, and Plural Quantification' (ms.) Forthcoming in Mind.

Yagisawa, Takashi. 'Possible Objects'. The Stanford Encyclopedia of Philosophy (Winter 2009 Edition), Edward N. Zalta (ed.), URL = $<$ http://plato.stanford.edu/archives/win2009/entries/possible-objects/ $>$.

Zalta, Edward. 'Essence and Modality'. Mind 115/459 (2006): 659-694. 